\title{
Punicalagin Inhibited Inflammation and Migration of Fibroblast-Like Synoviocytes Through NF-kB Pathway in the Experimental Study of Rheumatoid Arthritis
}

\author{
Mingcheng Huang ${ }^{1}, *$ \\ Keping $\mathrm{Wu}^{\mathrm{l}}$,* \\ Shan Zeng ${ }^{2, *}$ \\ Wenfen Liu' \\ Tianjiao Cui (D) \\ Zhiqing Chen $\mathbb{D}^{\prime}$ \\ Lian Lin' \\ Dongying Chen ${ }^{3}$ \\ Hui Ouyang'
}

'Department of Nephrology, Kidney and Urology Center, The Seventh Affiliated Hospital of Sun Yat-Sen University, ShenZhen, Guandong, People's Republic of China; ${ }^{2}$ Department of Rheumatology, The First Affiliated Hospital of Jinan University, Guangzhou, Guandong, People's Republic of China; ${ }^{3}$ Department of Rheumatology, The First Affiliated Hospital of Sun Yat-Sen University, Guangzhou, Guandong, People's Republic of China

*These authors contributed equally to this work
Correspondence: Dongying Chen; Hui Ouyang

Email chendy3@mail.sysu.edu.cn;

ouyh23@mail.sysu.edu.cn
Background: The aggressive phenotype of fibroblast-like synoviocytes (FLSs) is essential in the synovitis and bone destruction in rheumatoid arthritis (RA). Punicalagin is a natural polyphenol extracted in pomegranate juice, which possesses antioxidant, anti-inflammatory and anti-tumor properties suggesting it may be a potent drug for RA therapy. However, there is paucity of information on its effect in RA.

Objective: To investigate the effects of punicalagin on synovial inflammation and bone destruction in RA.

Methods: FLSs were isolated from synovial tissue of RA patients. The mRNA levels were evaluated by quantitative real-time PCR. Western blot was used for protein level measurements. The secretion of pro-inflammatory cytokines and metalloproteinases (MMPs) was detected by ELISA assays. Edu staining assays were carried out to investigate the proliferation of FLSs. Cell migration was assessed by Boyden chambers, wound scratch assays and F-actin staining in vitro. The intracellular translocation of nuclear factor kappa B (NF- $\kappa \mathrm{B})$ was investigated using immunofluorescence. The effects of punicalagin in vivo were measured by using collagen-induced arthritis (CIA) mice.

Results: Punicalagin treatments significantly reduced the TNF- $\alpha$ induced expressions of proinflammatory cytokines (IL-1 $\beta$, IL-6, IL-8 and IL-17A) and MMPs (MMP-1 and MMP-13) of RA FLSs. Punicalagin also suppressed the proliferation and migration of RA FLSs. Moreover, punicalagin $(50 \mathrm{mg} / \mathrm{kg} / \mathrm{d})$ alleviated arthritis severity and bone destruction, and decreased serum IL-6 and TNF- $\alpha$ in CIA mice. Further mechanism studies indicated that punicalagin blocked $\mathrm{NF}-\mathrm{\kappa} B$ activation via suppressing phosphorylation of IKK and $\mathrm{IkB} \alpha$, and preventing the translocation of 65 .

Conclusion: Our findings suggested that punicalagin might be one of natural therapeutic compounds for relieving RA progress via suppressing FLSs inflammation and migration through modulating NF- $\kappa$ B pathways.

Keywords: rheumatoid arthritis, punicalagin, fibroblast-like synoviocytes, migration, proinflammatory cytokines

\section{Introduction}

Rheumatoid arthritis (RA) is a chronic autoimmune disease featured by synovitis and hyperplasia, as well as bone destruction. Fibroblast-like synoviocytes (FLSs) play pivotal roles in pathophysiological process of synovial inflammation and bone destruction. ${ }^{1}$ RA FLSs can secrete high concentrations of pro-inflammatory 
cytokines, such as interleukin-1 $\beta$ (IL-1 $\beta)$, IL-6 and tumor necrosis factor (TNF- $\alpha$ ). Moreover, activated RA FLSs also exhibit tumor-like characters, such as vigorous proliferation, aggressive migration and high invasion, which are of critical importance in pannus development, cartilage and bone destruction. ${ }^{2}$ Therefore, modulating the inflammation, inhibiting proliferation and migration of RA FLSs is potential therapeutic strategy in treatments of RA. ${ }^{3}$

It has been recently reported that the intakes of polyphenol enriched fruits have beneficial effects in diabetes, cerebrovascular diseases, tumors, and arthritis. ${ }^{4,5}$ Pomegranate is the common fruit in Asian for hundreds of years. It is famous as "fruit of healing" as eating this fruit has beneficial effects in multiple diseases. Punicalagin is the most enriched polyphenols in pomegranate, which offer effects in medicine, such as antioxidant, anti-inflammatory, and anti-cancer effects. ${ }^{6,7}$ Recent researches revealed that punicalagin treatments reduced the lipopolysaccharide (LPS)-induced inflammatory responses in macrophage-derived RAW264.7, astrocytes and microglial BV-2 cells. ${ }^{89}$ Punicalagin has been shown to suppress proliferation in various cell lines, such as human oral tumor cells, colon and prostate tumor cells. ${ }^{10,11}$ Moreover, punicalagin inhibited migration and invasion of MCF-7 cells, ${ }^{12}$ osteosarcoma cells and human A2780 ovarian cancer cells in vitro ${ }^{12}$. Interestingly, punicalagin also had been reported to reduce the expression of matrix metalloproteinases (MMP). ${ }^{13,14}$

The above studies suggest punicalagin are potentially useful in the treatments of chronic inflammatory diseases and tumors. However, to date, the potential effects of punicalagin in RA are not clear. For this reason, we investigated the effects of punicalagin in collagen-induced arthritis (CIA) mice. We also investigated the potential effects of punicalagin in pro-inflammatory cytokines and MMPs production, cell proliferation, migration and its underlying mechanisms, with hope to provide new basis for the nutritional preventive strategy of RA.

\section{Materials and Methods}

\section{Patients}

A total number of 12 patients with active RA (10 female and 2 male, aged from 42 to 63 years-old) were recruited for this study. The diagnosis of RA was based on the 1987 revised criteria for American College of Rheumatology (ACR) ${ }^{15}$ Active RA was defined as disease activity score in 28 joints and four variables including $\mathrm{C}$-reactive protein
(DAS28-CRP) $\geq 3.2$. The demographics of RA patients are shown in Supplemental Table 1. Synovial tissues were obtained from active RA patients undergoing synovectomy or joint replacement.

\section{Ethical Approval}

All experimental procedures in this work were carried out according to the Declaration of Helsinki and approved by the Clinical Research Ethics Committee of the First Affiliated Hospital of Sun Yat-sen University (SYSU) University. Informed consent was obtained from every patient before the recruitment. Animal handling and procedures were approved by the Animal Care and Ethics Committee of SYSU and complied with the Guide for the Care and Use of Laboratory Animal published by the US National Institutes of Health (NIH publication, Eighth edition, 2011).

\section{Reagents and Antibodies}

For cell culturing, Dulbecco's Modified Eagle Medium F12 (DMEM/F12) cell culture mediums, fetal bovine serum (FBS), penicillin/streptomycin reagent, trypsin ethylenediaminetetraacetic acid (EDTA) and phosphate buffer saline (PBS) were supplied by Invitrogen (Carlsbad, CA, USA). TNF- $\alpha$ for in vitro studies was purchased from R\&D Systems (Minneapolis, MN, USA). Punicalagin was obtained from MedChemExpress (MCE) (USA).

\section{Cell Culture}

The FISs were isolated from individual patients immediately after sample collection, as we described previously. ${ }^{16}$ Briefly, freshly harvested synovium tissue was cut into small pieces, then digested for $2 \mathrm{~h}\left(37^{\circ} \mathrm{C}\right)$ using collagenase in DMEM/F12 medium. Human RA synoviocytes were cultured in DMEM/F12 medium (10\% FBS, $100 \mathrm{U} /$ $\mathrm{mL}$ penicillin and $100 \mathrm{mg} / \mathrm{mL}$ streptomycin) at $37^{\circ} \mathrm{C}$ in an incubator with $5 \% \mathrm{CO}_{2}$. All RA FLSs used in this study were between passages 3 and 6 .

\section{Methyl-Thiazol Tetrazolium Cytotoxicity Assay}

RA FLSs were cultured with or without Punicalagin $(12.5 \mu \mathrm{M}-100 \mu \mathrm{M})$ for $48 \mathrm{~h}$. The supernatant of the cell cultures were then replaced by medium containing methyl-thiazol tetrazolium (MTT, Sigma-Aldrich) and incubated for $0.5 \mathrm{~h}$. The resulting formazan was resuspended using acidified isopropanol and quantified at $570 \mathrm{~nm}(620 \mathrm{~nm}$ as reference). 


\section{EdU Proliferation Assay}

The effects of punicalagin on proliferation of RA FLSs were measured by 5-ethynyl-2-deoxyuridine (EdU) assay. Cells were treated with different concentrations of punicalagin $(12.5,25$ and $50 \mu \mathrm{M})$ for $24 \mathrm{~h}$. Then, cells were plated into 96 -well cell culturing plates at $1 \times 10^{4}$ cells/ well and cultured for $24 \mathrm{~h}$ in serum-free medium. After starving, the cells were incubated with $50 \mu \mathrm{M}$ EdU for $8 \mathrm{~h}$. EdU incorporation was assessed in triplicate using a Cell-Light ${ }^{\mathrm{TM}}$ EdU Apollo ${ }^{\circledR} 488$ in vitro Imaging Kit (Ribobio, Guangzhou, China) following the manufacturer's protocol. The experiment was independently repeated three times.

\section{Western Blot Analysis}

The method of Western blotting analyses was performed as described previously. ${ }^{17}$ Bicinchoninic acid (BCA) protein assay kit (Pierce, Rockford, IL, USA) was used to detect protein concentrations. Equal amounts of protein were solubilized in Laemmli buffer ( $\mathrm{pH}$ 6.8, $62.5 \mathrm{mM}$ Tris-HCl, 10\% glycerol, 2\% SDS, 5\% $\beta$-mercaptoethanol, and $0.0625 \%$ o bromophenol blue), boiled for 5 minutes, separated by $12 \%$ SDS-PAGE, and transferred to nitrocellulose membranes (EMD Millipore, Billerica, MA, USA). The membranes were probed with the following primary antibodies:

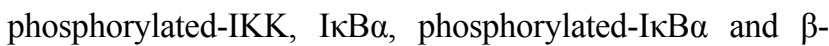
actin (all from Cell Signaling Technology, Danvers, MA, USA) in TBS/Tween-20 containing 5\% non-fat milk at $4{ }^{\circ} \mathrm{C}$ overnight. The membranes were incubated with the secondary antibodies for $1 \mathrm{hr}$ at room temperature. A peroxidaseconjugated secondary antibody (1:5000 dilution, BOSTER Biological Technology Co. Ltd, Wuhan, China) and enhanced chemiluminescence Western blot detection reagents (NCI4106, Pierce, ThermoFisher) were used to visualize the target proteins. Each blot is representative of at least 3 independent experiments. The film was scanned by a high-resolution scanner, densitometry was performed using AlphaEaseFc software on a Fluorchem 8900 system (Alpha Innotech, San Leandro, CA, USA).

\section{ELISA Detection of Pro-Inflammation Cytokines and MMPs in Culture}

\section{Supernatant}

ELISA assay was performed to detect verify IL-1 $\beta$, IL-6, IL-8, IL-17A, MMP-1 and MMP-13 in culture supernatant by using commercial kits (R\&D Systems, Minneapolis, MN, USA). As briefly, prepare controls, samples and working standards, add $100 \mu \mathrm{L}$ of Assay Diluent RD1W to each well. Then add $100 \mu \mathrm{L}$ of standard, control, or samples per well. Cover with the adhesive strip and incubate for $2 \mathrm{~h}$ at room temperature. Aspirate each well and wash for 3 times. Wash by filling each well with Wash Buffer $(400 \mu \mathrm{L})$ using a squirt bottle, manifold dispenser, or auto-washer. After the last wash, remove any remaining Wash Buffer by aspirating or decanting. Invert the plate and blot it against clean paper towels. Add $200 \mu \mathrm{L}$ of human IL-1 $\beta$, IL-6, IL-8, IL-17A, MMP-1 and MMP-13 conjugate to each well, respectively. Cover with a new adhesive strip and incubate for $2 \mathrm{~h}$ at room temperature. Aspirate each well and wash for 3 times. Add $200 \mu \mathrm{L}$ of substrate solution to each well and incubate for 20 minutes at room temperature. Add $50 \mu \mathrm{L}$ of stop solution to each well. The color in the wells should change from blue to yellow. Determine the optical density of each well within 30 minutes, using a microplate reader set to $450 \mathrm{~nm}$. The culture supernatant was tested in triplicate.

\section{Quantitative Real-Time PCR Analysis}

Total RNA was isolated by TRIzol reagent (Invitrogen, San Diego, CA, USA) according to the manufacturer's protocol. Purified RNA samples were reverse transcribed with a PrimeScript ${ }^{\mathrm{TM}}$ RT reagent kit (Takara, Dalian, China) according to the manufacturer's instructions. IL-1 $\beta$, IL-6, IL-8, IL-17A, MMP-1 and MMP-13 mRNA levels were determined by real-time PCR using CFX384 Real-Time PCR Detection System (Bio-Rad, Hercules, CA, USA) and SYBR Green qPCR Master Mix (Takara). Primers are listed in Supplemental Table 2, and $\beta$-actin was used as a quantitative control for RNA levels. To quantify the relative expression of each gene, $\mathrm{Ct}$ values were normalized to the endogenous reference $(\Delta \mathrm{Ct}=\mathrm{Ct}$ target- $\mathrm{Ct} 18 \mathrm{~S}$ rRNA) and compared with a calibrator using the $\Delta \Delta \mathrm{Ct}$ method $(\Delta \Delta \mathrm{Ct}=\Delta \mathrm{Ct}$ sample- $\Delta \mathrm{Ct}$ calibrator $)$.

\section{Transwell Assays for Vertical Migration}

The effects of punicalagin on vertical migration RA FLSs were detected using transwell assays according to the method previously described. ${ }^{18}$ In short, DMEM containing TNF- $\alpha$ (10ng/mL, R\&D Systems, Minneapolis, Minnesota, USA) as a chemoattractant was placed in the lower well. RA FLSs (final concentration $6.0 \times 10^{4}$ cells $/ \mathrm{mL}$ ) were suspended in serum-free DMEM in the upper well. The chamber was incubated at $37^{\circ} \mathrm{C}, 5 \% \mathrm{CO}_{2}$ for $8 \mathrm{~h}$. After incubation, use a cotton swab to remove non-migrating cells from the upper 
surface of the filter. The filter was fixed in methanol for 15 min and stained with $0.1 \%$ crystal violet for $15 \mathrm{~min}$. Chemotaxis was quantified by counting the stained cells that migrated to the underside of the filter using an optical microscope (magnification 100×). For each assay, the stained cells were counted as the average number of cells per 10 random fields. Assays were performed in triplicate.

\section{Wound Healing Assay for Horizontal Migration}

The effects of punicalagin on the horizontal migration of RA FLSs were detected by wound healing assay. ${ }^{18}$ RA FLSs were seeded into 6-well plates to almost total confluence. An artificial homogenous wound was created onto the monolayer with a sterile $10-\mu \mathrm{L}$ tip. After scratching, the culture dishes were washed with serum-free medium. Images of cells migrating into the wound were captured by an inverted microscope $(\times 100)$, and the area of scratch was measured by ImageJ 1.47 analysis system (National Institutes of Health, Bethesda, MD, USA).

\section{F-Actin Staining for Reorganization of Actin Cytoskeleton}

The effect of punicalagin on regulating RA FLSs actin cytoskeleton reorganization was detected by F-actin staining. RA FLSs were seeded on sterilized glass coverslips in 35-mm dishes. The cells were stimulated with punicalagin for $24 \mathrm{~h}$, then they were fixed with paraformaldehyde and infiltrated with $0.3 \%$ Triton X-100 in PBS. To detect F-actin, the cells were incubated with phalloidin overnight. The cells were then incubated with DAPI and the cover slips were mounted on glass slides with anti-fade mounting media, and the confocal fluorescence microscope (LSM 710, Zeiss, Germany) was used for inspection.

\section{CIA Mice and Treatments}

Based on the reported method, ${ }^{19,20}$ DBA/1 mice (male, 8-9 weeks, SLAC Laboratory Animal Company, Shanghai, China) were intradermally injected at the tail base with $200 \mathrm{mg}$ of bovine type II collagen emulsified at a 1:1 ratio (vol/vol) in Freund's complete adjuvant (day 0), and then boosted 21 days later using bovine type II collagen emulsified at a 1:1 ratio in incomplete Freund's adjuvant (day 21). CIA mice were randomly treated daily by intraperitoneal injection of DMSO $(n=8)$ or punicalagin $(50 \mathrm{mg} / \mathrm{kg} / \mathrm{d}, \mathrm{n}=8)$ for 14 days, which was initiated on the second day of booster immunization (day 22). We also set up a normal control group including 8 mice without any treatment.

\section{Assessment of Clinical Scores and Paw Thickness}

When the symptoms of arthritis appeared, the rats were monitored every other days for signs of arthritis. The arthritis severity was scored on a scale as follows: 0 , normal joints; 1, swelling and redness of at least 1 joint; $2=$ swelling in $>1$ joint; 3 , moderate swelling in the entire paw; and 4, joint deformity and/or ankylosis. ${ }^{21}$ The total clinical score was recorded as the sum for all 4 limbs. Swelling was quantified by measuring the thickness of the first arthritic hind paw with a caliper in all groups.

\section{Assessment of Pathologic Changes in $\mathrm{CIA}$ Joints}

After punicalagin (or DMSO) treatments for 14 consecutive days, samples of the hind limbs were freshly collected and kept in $10 \%$ formalin for $48 \mathrm{~h}$ for fixation. After a series of dehydration, waxing and embedding, the joints were cut into 5 micrometer $(\mu \mathrm{m})$-thick slices. The slices were stained by hematoxylin for 5 minutes, then rinsed with running water. Next, the slices were stained with eosin solution for 1 3 $\mathrm{min}$, then wash with water. Images of specimens were captured under microscope. Level of inflammatory response, hyperplasia and bone damages were scored as follow: 0, no symptom shown; 1, mild; 3, moderate; and 4, severe. All scoring was performed by two blinded pathologists.

\section{Assessment of Serum IL-6 and TNF- $\alpha$ in CIA Mice}

Mice plasma was obtained by the centrifugation of blood in serum separator Microtainer tubes after cardiac puncture. ELISA assay was used to detect serum IL-6 and TNF- $\alpha$. The tested serum, standards and the negative control were incubated at $37^{\circ} \mathrm{C}$ for $30 \mathrm{~min}$, and then washed with PBS for 5 times. Add the enzyme-labeled reagent and incubated for $2 \mathrm{~h}$ at room temperature, then washed for 5 times. Next, add $0.05 \%$ TMB solution developing solution A, B. Enter the stop solution to stop the reaction, detect in a $450 \mathrm{~nm}$ microplate reader, read and calculate OD value. 


\section{Detection of Serum Biochemical Parameters of $\mathrm{CIA}$}

Serum aspartate aminotransferase (AST) was tested by AST reagent kit (Rate method), serum alanine aminotransferase (ALT) was examined by ALT reagent kit (Rate method), Serum creatinine (CR) was tested by CRE reagent kit (Enzymatic method). All the measurements were strictly performed according to the manufacturer's instructions (NanJing JianCheng Bioengineering Institute).

\section{Statistical Analysis}

Data were presented as mean \pm Standard Error of Mean (SEM) for continuous variables and presented as frequencies and percentages for categorical variables. Comparison between groups was evaluated with chi-square test or Fisher exact test for categorical variables. Comparison between groups was evaluated with 2-tailed student $t$ test or one-way ANOVA followed by Bonferroni's post hoc comparisons for continuous variables with normal distribution. Between-group comparison was evaluated using the Mann-Whitney $U$-test for continuous variables with nonnormal distribution. A $P$ value of less than 0.05 was considered significant. Statistical analyses of the data were performed using SPSS, version 13.0 (IBM).

\section{Results}

\section{The Effects of Punicalagin on Cell Viability}

Firstly, we use MTT staining to explore the effects of punicalagin on cell viability of RA FLSs. Punicalagin significantly inhibited cell viability at concentration of $100 \mu \mathrm{M}$ when compared with non-treated cells $(P<0.05$, Figure 1). Punicalagin at the low concentration $(12.5,25$,

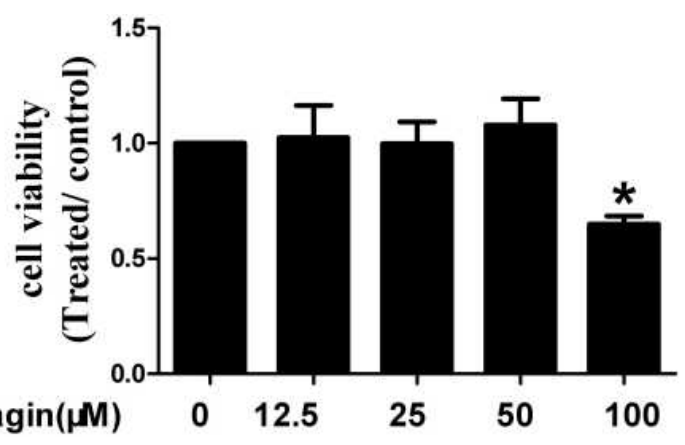

Figure I Effects of punicalagin on cell viability in RA FLSs. Cell viability was tested by MTT assays in RA FLSs incubated with punicalagin (at the concentration 12.5, 25, and $50 \mu \mathrm{M}$ ) for $48 \mathrm{~h}$. Data are means of three separated experiments \pm SEM. $* P<$ 0.05 , vs control.

Abbreviations: RA, rheumatoid arthritis; FLSs, fibroblast-like synoviocytes; MTT, methyl-thiazol tetrazolium cytotoxicity.
$50 \mu)$ has no effect on cell viability. In the subsequent experiments, we choose the punicalagin concentrations $(12.5,25,50 \mu \mathrm{M})$ that did not affect the cell viability.

\section{Punicalagin Inhibits TNF- $\alpha$ Induced Pro- Inflammatory Cytokines Production}

In order to mimic the local inflammatory microenvironment of the synovium in RA, $10 \mathrm{ng} / \mathrm{mL}$ of TNF- $\alpha$ was added to stimulate RA FLSs for $24 \mathrm{~h}$. Under this condition, punicalagin was able to decrease the mRNA levels of pro-inflammatory cytokines (IL-1 $\beta$, IL-6, IL-8 and IL$17 \mathrm{~A}$ ) induced by TNF- $\alpha$ in RA FLSs (Figure 2A-D). Moreover, punicalagin also decreased the concentration of IL-1 $\beta$, IL-6, IL- 8 and IL-17A in the supernate (Figure $3 \mathrm{~A}-\mathrm{D})$. These results indicated that punicalagin inhibited TNF- $\alpha$ induced pro-inflammatory cytokine production in RA FLSs.

\section{Punicalagin Inhibits Cell Proliferation of RA FLSs}

Aberrant proliferation of FLSs plays a key role in the process of synovial hyperplasia and pannus formation. Thus, the effects of punicalagin on the cell proliferation of RA FLSs were studied. Punicalagin inhibited the proliferation of RA FLSs dose-dependently (Figure 4A-B). Above results showed that punicalagin exerted an inhibitory effect on the cell proliferation of RA FLSs.

\section{Punicalagin Inhibits in vitro Migration of RA FLSs}

Subsequently, we evaluated the functions of punicalagin in the migrations of RA FLS by Transwell chamber assay. The results showed that treatment with punicalagin markedly suppressed migrations of RA FLSs in a dose-dependent manner (Figure 5A). Then, we use wound healing assays to confirm the effect of punicalagin on the migration ability. The results showed that punicalagin significantly inhibited RA FLSs migrate into the wounded area (Figure 5B). Dynamic reorganization of the actin cytoskeleton is critical for RA FLSs migration. Therefore, we investigated whether punicalagin inhibited the reorganization of the actin cytoskeleton. As shown in Figure 5C, treatment with punicalagin inhibited actin stress fiber formation. The above indicated that punicalagin controlled RA FLSs migration might be via regulating the reorganization of cytoskeleton. 

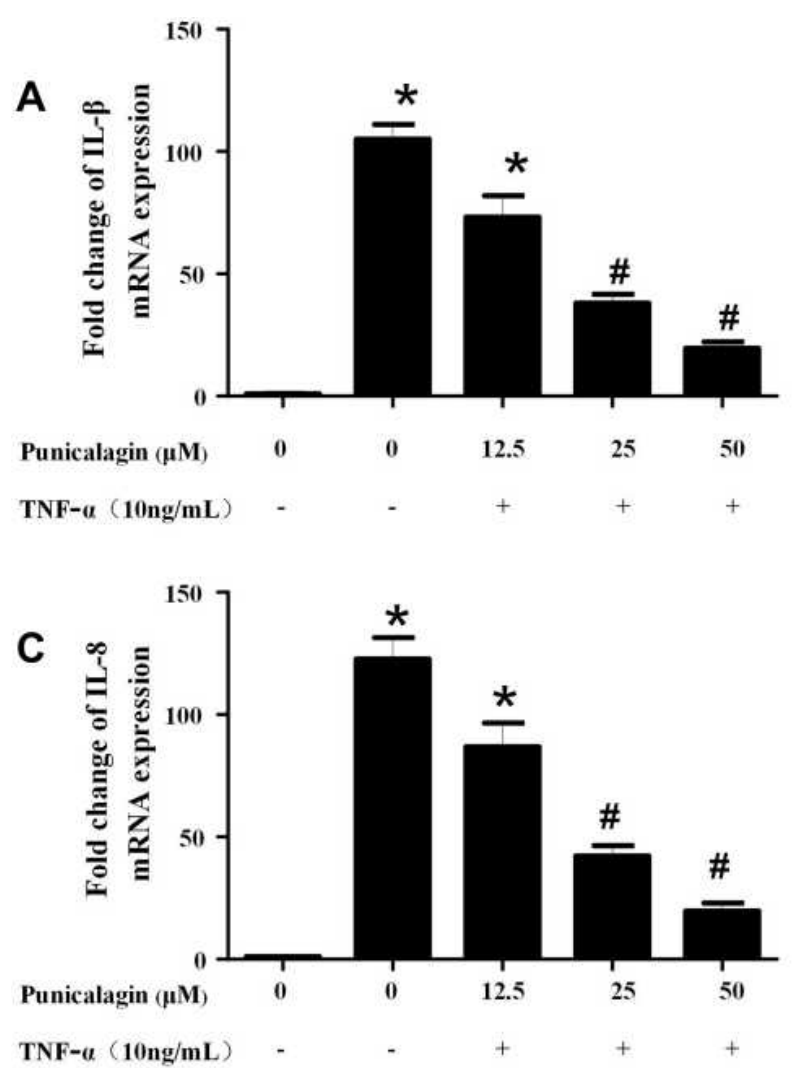
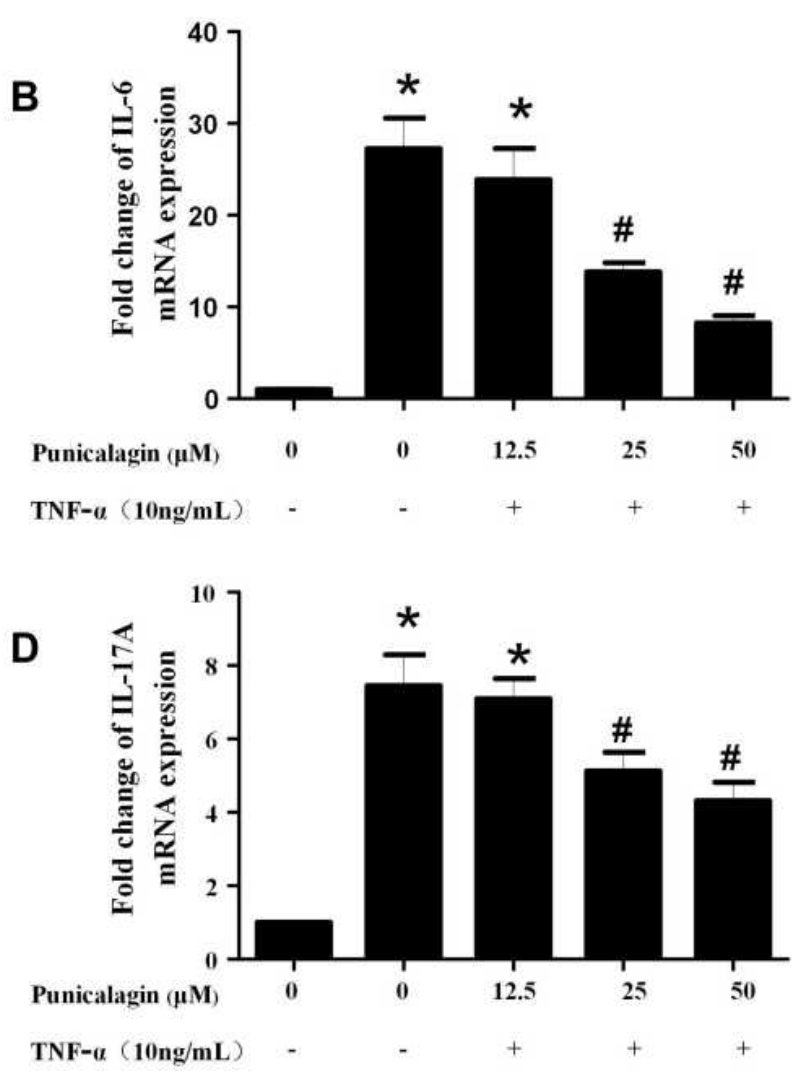

Figure 2 Punicalagin decreased the mRNA expression of pro-inflammatory cytokines in RA FLSs. RA FLS were stimulated with TNF- $\alpha$ (IOng/mL) for I $2 \mathrm{~h}$ in the presence or absence of punicalagin (at the concentration 12.5, 25, and 50 $\mu \mathrm{M}$ ). Quantitative real-time PCR was used to measure the levels of mRNA expression. A-D, the expression of mRNA levels of IL-I $\beta$, IL-6, IL-8 and IL-I7A, respectively. Data were normalized to $\beta$-Actin. Data represent the mean \pm SEM of 3 independent experiments. $* P<0.05$ vs control, ${ }^{\#} P<0.05$ vs TNF- $\alpha$.

Abbreviations: TNF, tumor necrosis factor; PCR, polymerase chain reaction; RA, rheumatoid arthritis; FLSs, fibroblast-like synoviocytes; IL, interleukin.

\section{Punicalagin Inhibits TNF- $\alpha$-Induced MMPs Production}

Activated RA FLSs can express high levels of MMPs, which play key roles in the destruction of cartilage and bone in RA. Next, we used qPCR and ELISA to detect the production of MMPs in RA FLSs. The result of qPCR verified that the mRNA expression of MMP-1 and MMP-13 in RA FLSs was significantly decreased in the punicalagin group compared with the untreated group (Figure 6A and B). This was confirmed again with ELISA showing significantly decreased secreted forms of MMP-1 and MMP-13 in the culture supernatant (Figure 6C and D). As we know, MMPs play irreplaceable roles in cell invasion, so the results indicated that punicalagin might inhibit in vitro invasion of RA FLSs by attenuating MMPs expression.

\section{Punicalagin Suppresses TNF- $\alpha$-Induced NF-kB Pathway Activation}

Nuclear Factor Kappa-B (NF-kB) is recognized as one of the main inflammatory pathways in RA, which exhibits high levels of pro-inflammatory cytokines linked to bone erosion and disease progression. ${ }^{22}$ Thus, we try to investigate the effects of punicalagin on the NF- $\kappa \mathrm{B}$ pathway activation. We found that phosphorylation of IKK and I $\mathrm{B} \alpha$ was markedly increased after TNF- $\alpha$ stimulation at $15 \mathrm{~min}$. Treatment of punicalagin $(50 \mu \mathrm{M})$ significantly decreased phosphorylated IKK $\beta$ and I $\mathrm{B} \alpha$ in TNF- $\alpha$-stimulated RA FLSs (Figure 7A).

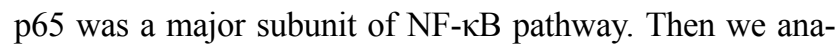
lyzed whether punicalagin affected p65 nuclear translocation. Immunofluorescence staining showed that TNF- $\alpha$ increased p65 translocation into the nucleus, and punicalagin significantly reduced the level of p65 in the nucleus (Figure 7B).

\section{Punicalagin Attenuates of the Severity of Arthritis in CIA Mice}

Next, we use CIA mice to investigate the in vivo effects of punicalagin in RA. Histological examinations indicated that mice received punicalagin had a reduction in pathological disease severity, as indicated by inflammatory cell infiltration, synovial hyperplasia and cartilage damage when 

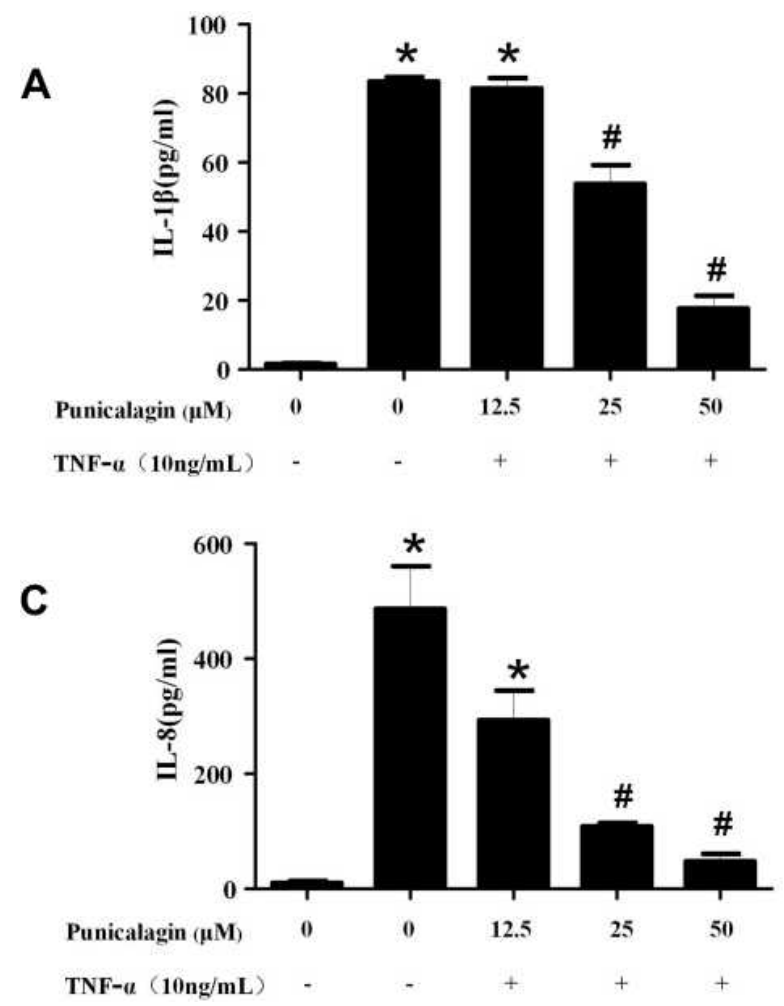

B
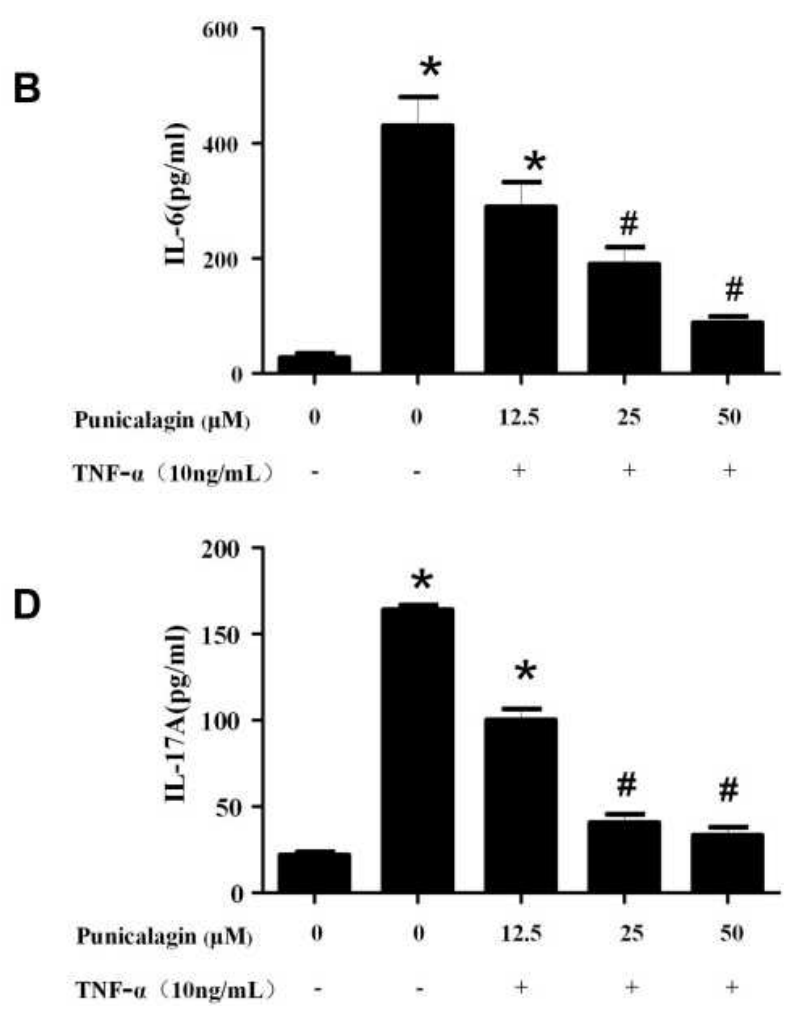

Figure 3 Punicalagin decreased the supernatant concentration of pro-inflammatory cytokines of RA FLS. RA FLS were stimulated with TNF- $\alpha$ (IOng/mL) for $24 \mathrm{~h}$ in the presence or absence of punicalagin (at the different concentration 12.5, 25, and 50 $\mu \mathrm{M}$ ). (A-D) The levels of pro-inflammatory cytokines (IL-I $\beta$, IL-6, IL-8 and IL-I7A) in supernatants of cell culturing medium were tested by ELISA assays. The data represent the mean \pm SEM of 3 independent experiments. $* P<0.05$ vs control, ${ }^{\#} P<0.05$ vs TNF- $\alpha$.

Abbreviations: RA, rheumatoid arthritis; FLSs, fibroblast-like synoviocytes; IL, interleukin; TNF, tumor necrosis factors.

compared to CIA controls (Figure 8A). Evaluation of clinically relevant endpoints showed that the clinical score and paw thickness in the punicalagin treated group $(50 \mathrm{mg} / \mathrm{kg} / \mathrm{d})$ were significantly less than the control group (Figure $8 \mathrm{~B}$ and C). To confirm the in vivo effect of punicalagin on the production of pro-inflammatory cytokines, we used ELISA assays to detect the serum levels of IL- 6 and TNF- $\alpha$ in CIA mice. We found that the serum levels of IL- 6 and TNF- $\alpha$ were markedly decreased by punicalagin (Figure $8 \mathrm{D}$ ). The peripheral blood of mice was obtained to examine the toxicity of CIA mice in vivo. The serum levels of ALT, AST and CRE in the punicalagin group were similar when compared to that of the control group (Figure 8E and F). These results suggested that punicalagin attenuated synovial inflammation and bone destruction of CIA mice. Moreover, punicalagin did not show any evidence of hepatocyte or glomerular injury as determined by serum biochemical parameter tests.

\section{Discussion}

Due to the high cost and the increasing risk of serious infections, the application of biologics is limited in clinical practice, especially in developing countries. Therefore, effective and safe treatments are urgently needed. Recent investigations provided evidences for the potential beneficial effects of natural polyphenols. It was demonstrated that polyphenol enriched foods produce protective effects against several chronic diseases, such as diabetes, ${ }^{23}$ Alzheimer's disease, ${ }^{24}$ and cardiovascular diseases. ${ }^{25}$ However, its effects on RA and its mechanism are not clear. In this study, we focused on polyphenols extracted from natural sources, and found a novel function of on inhibition of inflammation and tumor-like properties of RA FLSs. Our results also suggested punicalagin blocked the TNF- $\alpha$-simulated activations of NF- $\mathrm{KB}$ signaling pathway. Treatments of punicalagin alleviated synovial inflammation and bone destruction in vivo without hepatocyte or glomerular injury. Collectively, our findings suggested that punicalagin was a potential treatment for RA.

RA synovium produces various of pro-inflammatory cytokines including TNF- $\alpha$, IL-1 $\beta$, IL-6, and IL-17A which is involved in synovial pannus formation, osteoclast activation and joint destruction via activation of macrophages, 
A

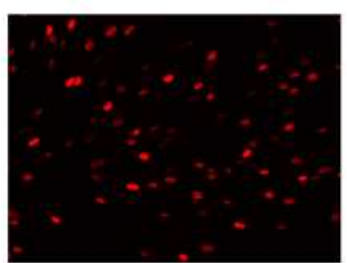

0

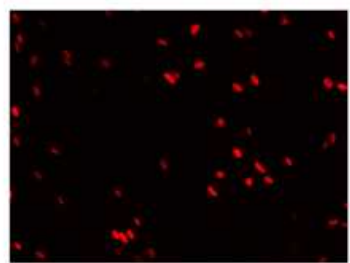

12.5

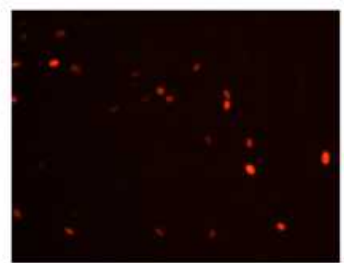

25

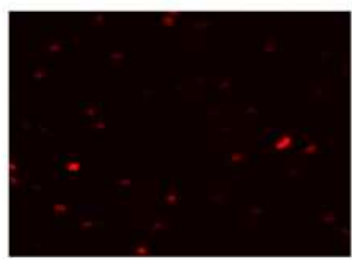

50

B

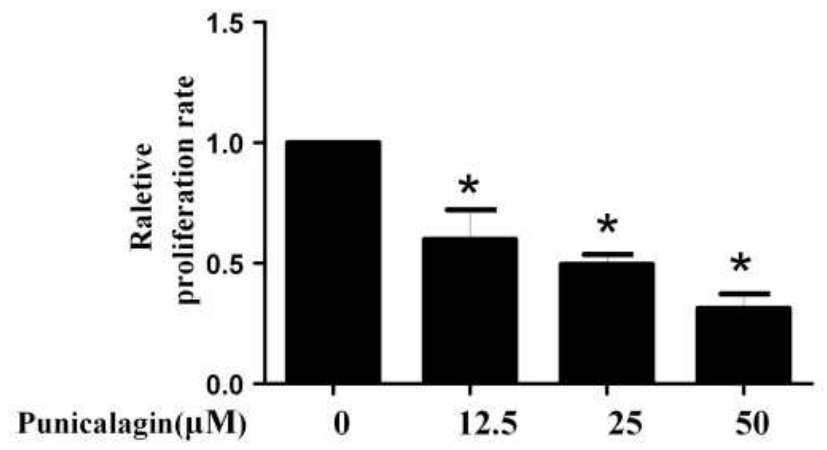

Figure 4 Punicalagin inhibited the cell proliferation of RA FLSs. (A) Cell proliferation was tested by EdU incorporation assays. Representative images show the proliferation of RA FLSs (A) labeled with EdU (red) (original magnification, $\times 200$ ). (B) The bar represents the relative proliferation rate of RA FLSs. Graphs in A indicated the mean \pm SEM of 3 independent experiments involving 6 different RA patients. $* P<0.05$ vs control.

Abbreviations: RA, rheumatoid arthritis; FLSs, fibroblast-like synoviocytes.

A Punicalagin

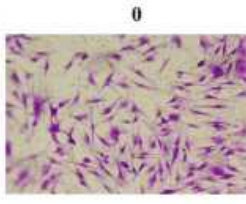

B

Punicalagin

0

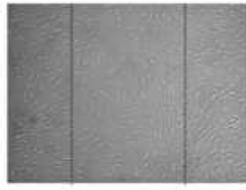

DMSo

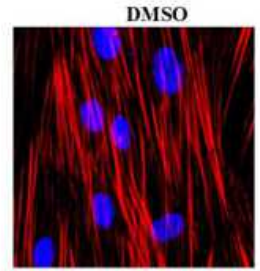

$12.5 \mu \mathrm{M}$

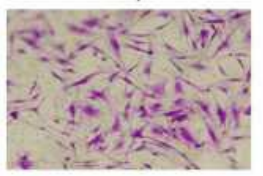

$12.5 \mu \mathrm{M}$

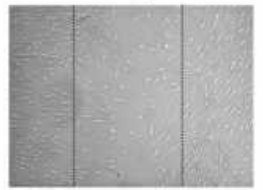

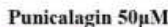

$25 \mu \mathrm{M}$

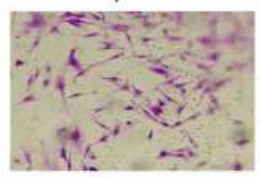

$25 \mu \mathrm{M}$

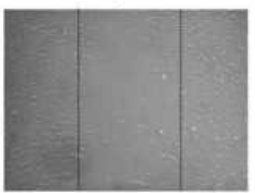

$50 \mu \mathrm{M}$

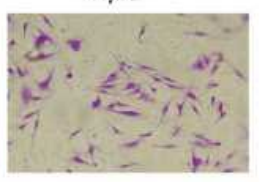

$50 \mu \mathrm{M}$

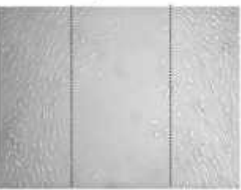

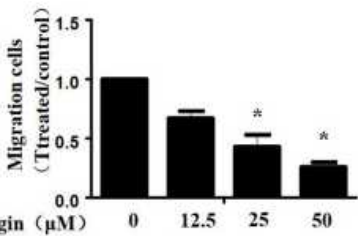

$\begin{array}{lllll}\text { Punicalagin }(\mu \mathrm{M}) & 0 & 12.5 & 25 & 50\end{array}$

C

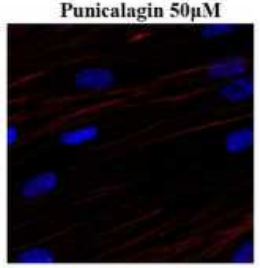

Figure 5 Punicalagin suppressed in vitro migration of RA FLSs. (A) Chemotaxic migration of RA FLSs was evaluated using a Transwell assay. Representative images (original magnification, $\times 100)$ are shown. Graphs indicate the relative migration rates. The relative migration rate represents the number of migrated cells normalized to the control. (B) The migration of RA FLSs was analyzed using a wound-healing assay. Representative images are shown (original magnification, $\times 50$ ). (C) Effect of punicalagin on the actin cytoskeleton reorganization of RA FLSs. F-actin (red) and nucleus (blue) were stained with phalloidin and DAPI, respectively. Representative images are shown (original magnification, $\times 100$ ). Data are shown as the mean \pm SEM of 3 independent experiments involving 5 different RA patients. $* P<0.05$ vs control.

Abbreviations: RA, rheumatoid arthritis; FLSs, fibroblast-like synoviocytes. 

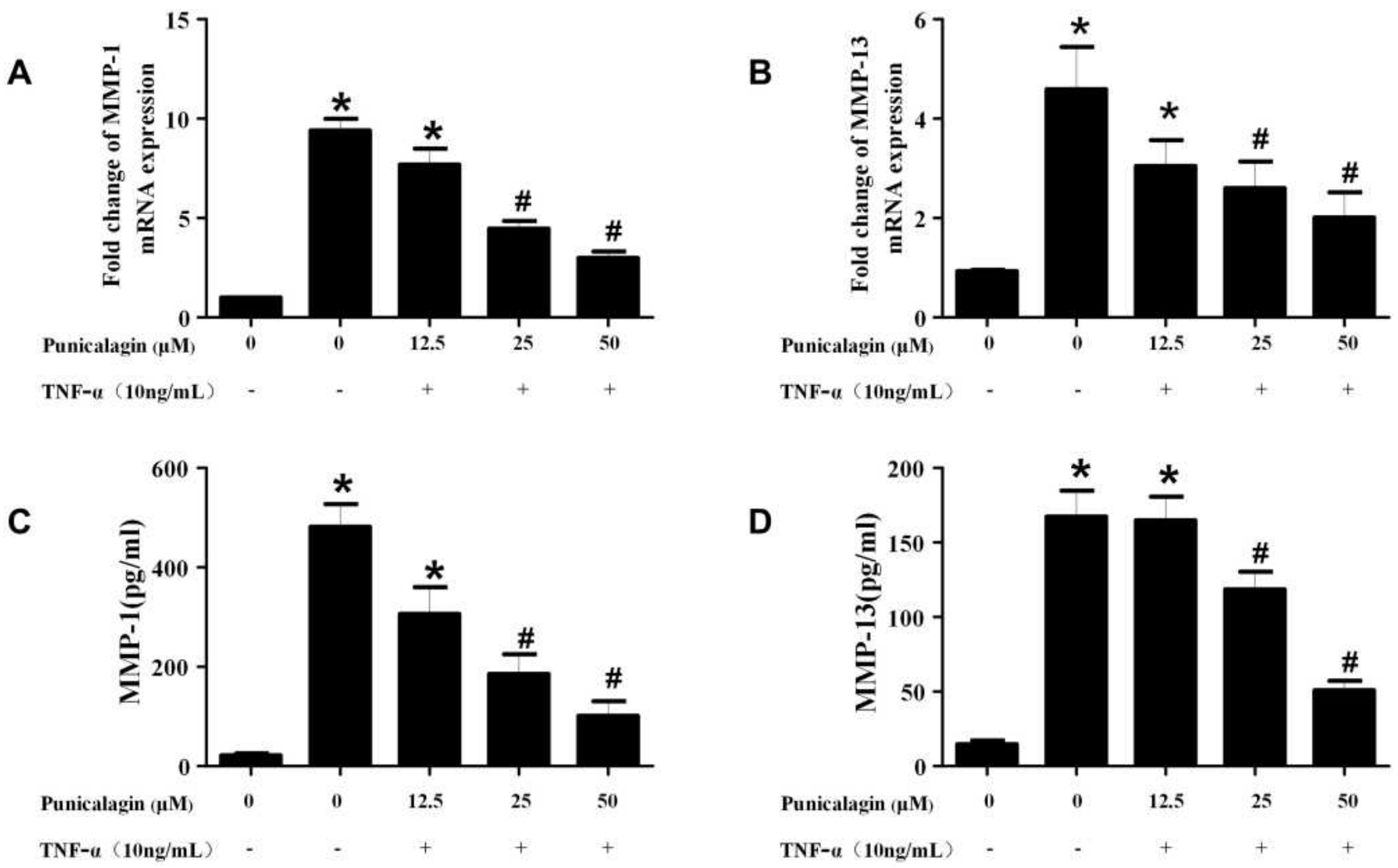

Figure 6 Effects of punicalagin on the production of MMPs in RA FLSs. (A-B) RA FLS were stimulated with TNF- $\alpha(10 \mathrm{ng} / \mathrm{mL})$ for $12 \mathrm{~h}$ in the presence or absence of

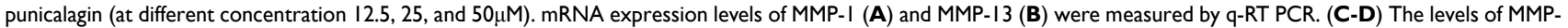
I (C), and MMP-I3 (D) secreted in culture media by TNF- $\alpha$ stimulated (IOng/mL for $24 \mathrm{~h})$ RA FLSs were measured with ELISA. Data represent the mean \pm SEM of 3 independent experiments. ${ }^{*} P<0.05$ vs control, ${ }^{\#} P<0.05$ vs TNF- $\alpha$.

Abbreviations: RA, rheumatoid arthritis; FLSs, fibroblast-like synoviocytes; MMPs, metalloproteinases.

FLSs, helper T (Th) cells and osteoclasts. ${ }^{26}$ In addition, IL-8 is an important chemokine, which promotes neutrophil chemotaxis to the inflammation site. ${ }^{27}$ With the promoting effects of pro-inflammatory cytokines, RA FLSs are able to release the chemokines and MMPs to enhance synovial inflammation and bone destruction. Recent in vitro studies indicate that punicalagin potently suppressed the production of pro-inflammatory cytokines in LPS-activated microglia and RAW264.7. ${ }^{8,28,29}$ Besides, in vivo researches reported that punicalagin attenuated ischemia/reperfusion injury and lung edema through inhibiting IL- 6 and TNF- $\alpha$ productions in mice. ${ }^{30,31}$ In addition, punicalagin significantly decreased protease-activated receptor-2-induced increases in IL-8, IFN- $\gamma$, and TNF- $\alpha$ in the human podocyte cell line. Notably, punicalagin significantly alleviated kidney injury in NZB/W F1 mice. The results suggest that punicalagin is a potential therapeutic agent for lupus nephritis. ${ }^{32}$ Another study also suggested the punicalagin exerted a protective role in ankylosing spondylitis via reducing oxidative stress and the production of inflammation cytokines by the Th17 cells and the IL-17A/IL-23 axis. $^{33}$ In our findings, we demonstrated that the treatments of punicalagin significantly down-regulated the expression and secretion of IL-1 $\beta$, IL-6, IL-8 and IL-17A. These findings make punicalagin a promising target for anti-inflammatory therapies of RA.

RA FLSs are the most common cell type that contribute to joint destruction through their production of cytokines, chemokines, and MMPs and by migrating and invading joint cartilage. Therefore, regulation the migration of RA FLSs might be a new treatment strategy for the joint destruction progress of RA. Recently, several researches demonstrated that punicalagin inhibited in vitro migration in many tumor cell lines, such as breast cancer cells, ${ }^{12}$ osteosarcoma cells, ${ }^{34}$ ovarian carcinoma cells, $^{35}$ and non-small cell lung cancer cells. ${ }^{14}$ Consistently, our findings suggested that punicalagin treatments inhibited migration of RA FLSs, revealed by both transwell assay and wound healing assay. Cell cytoskeleton rearrangement plays an essential role in regulating the direction of cell migration and invasion. Our study demonstrated that punicalagin prevented F-actin remodeling in RA FLSs. All of the results indicated punicalagin inhibited 


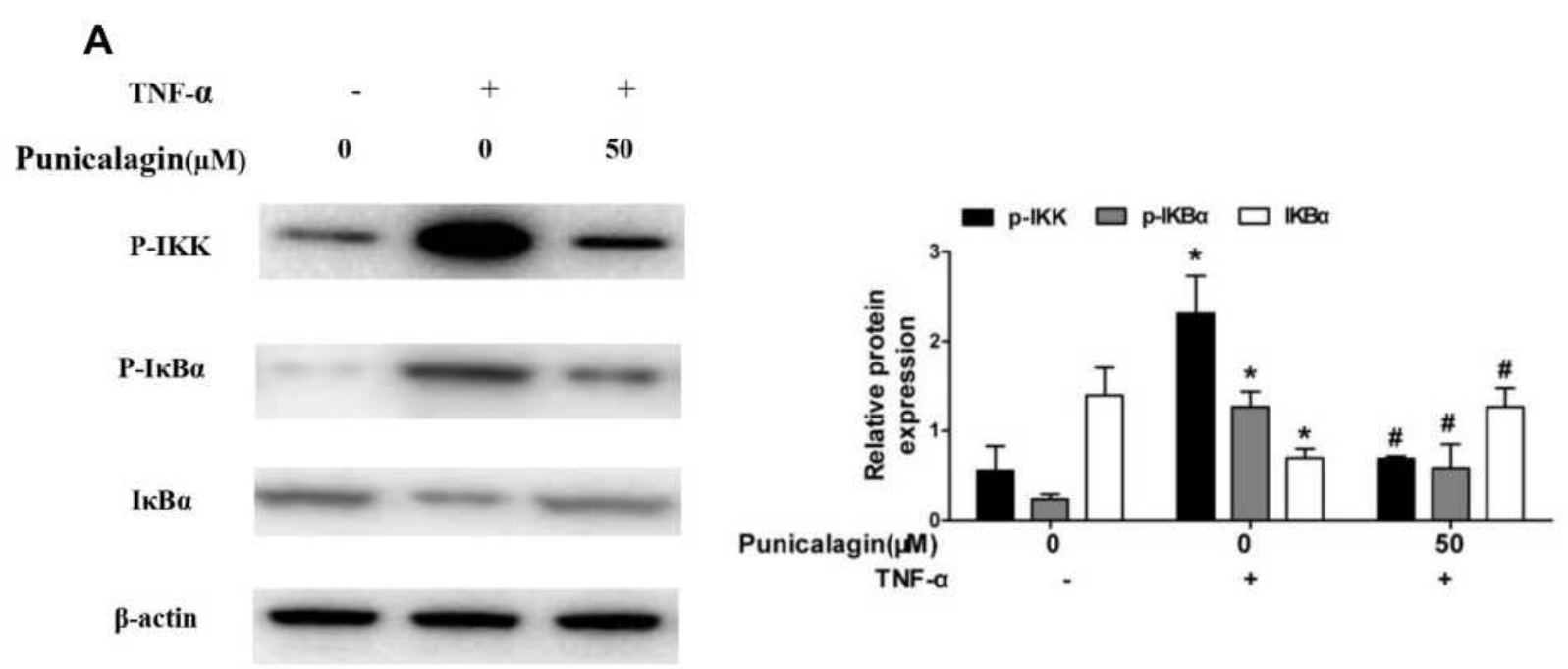

B

DAPI

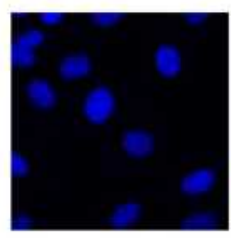

DMSO

TNF $\alpha+D M S O$
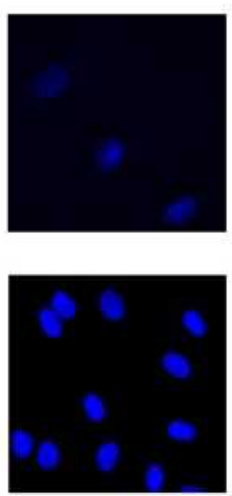

P65
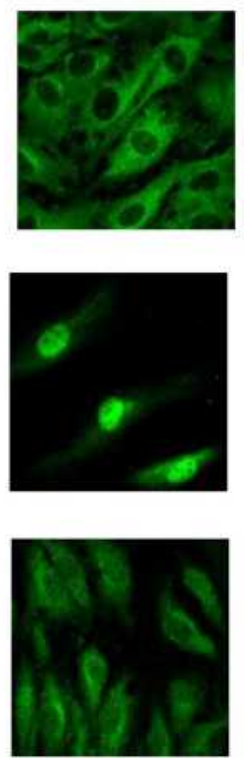

MERGE
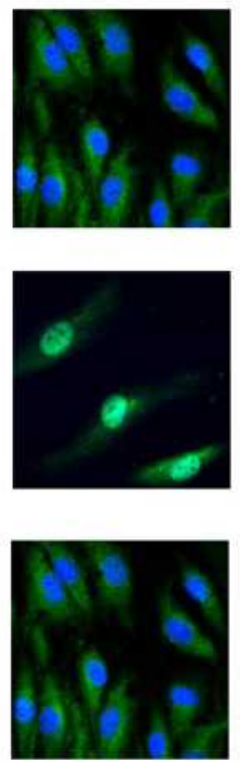

Figure 7 Punicalagin inhibits TNF- $\alpha$ induced NF- $\kappa$ B pathway activation and $\mathrm{p} 65$ relocation. (A) RA FLSs were pre-incubated with punicalagin for $24 \mathrm{~h}$ and TNF- $\alpha$ ( $10 \mathrm{ng} / \mathrm{mL}$ ) for $15 \mathrm{~min}$. Phosphorylation of IKK and IKB $\alpha$ induced by TNF- $\alpha$ was analyzed by Western blot analysis. The right panel shows a densitometric analysis of Western blots. The experiment was repeated at least three times. Each bar represents mean \pm SD from 6 RA patients. $* P<0.05$ vs control, ${ }^{\#} P<0.05$ vs TNF- $\alpha$. (B) Immunofluorescence staining analysis of p65 localization. RA FLSs were pre-incubated with $50 \mu \mathrm{M}$ punicalagin for $24 \mathrm{~h}$ and TNF- $\alpha$ (IOng/mL) for $15 \mathrm{~min}$, fixed, and then underwent immunofluorescence assay. The cells were stained with anti-p65 (in green) and DAPI (in blue). Representative 200× images.

in vitro migration of RA FLSs. Many previous studies have shown that the high expression of MMPs in RA FLSs were essential to cell invasion and collagen degradations. ${ }^{36}$ In our study, we found punicalagin decreased the production of both MMP-1 and MMP-13. This finding was consistent with the previous finding in human PM10-exposed keratinocytes. ${ }^{37}$ More interestingly, it has been reported that punicalagin had an effect of ameliorating mitigate osteoarthritis-induced cartilage erosion. $^{38}$

$\mathrm{NF}-\mathrm{\kappa B}$ is a transcription factor that participates in regulating the expression of pro-inflammatory genes. In RA, activated NF-KB pathway was a key regulator in the development of synovial inflammation and joint destruction. ${ }^{39}$ $\mathrm{NF}-\mathrm{\kappa B}$ activation could be modulated at multiple steps. In this study, we observed that punicalagin suppressed the TNF- 
A

Normal
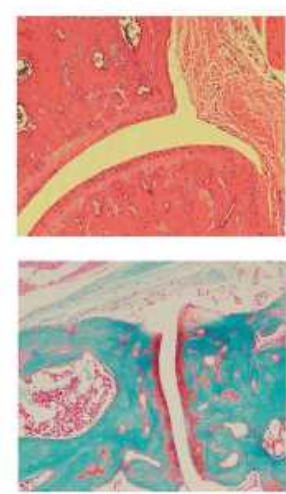

B

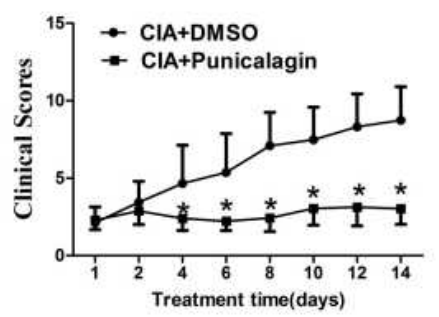

D

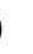

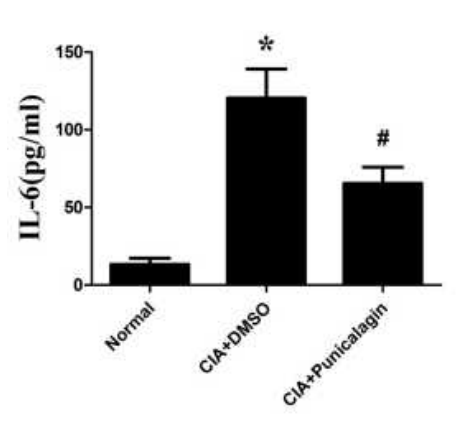

E

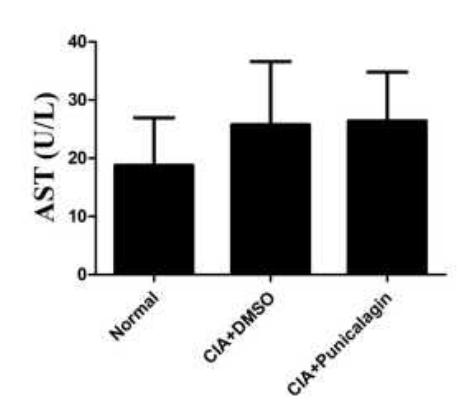

CIA+DMSO
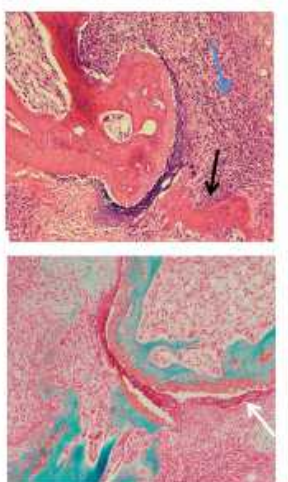

\section{C}

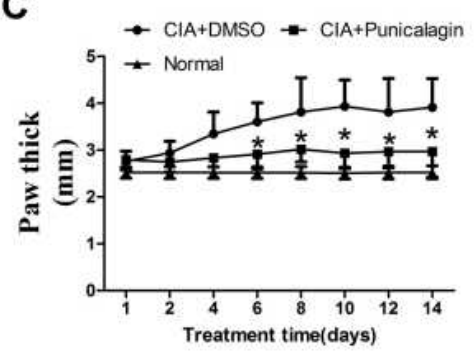

CIA+Punicalagin

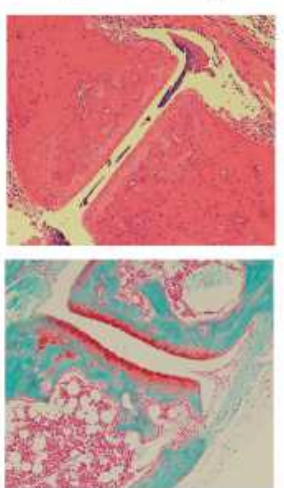

(4)

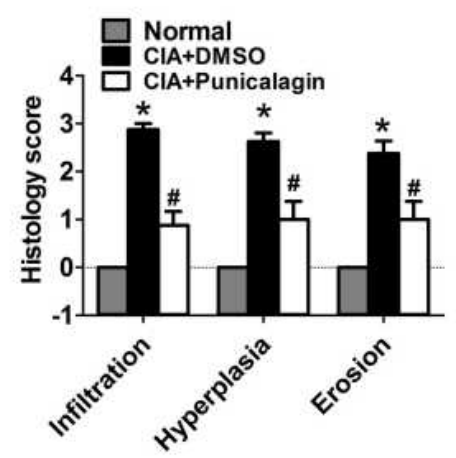

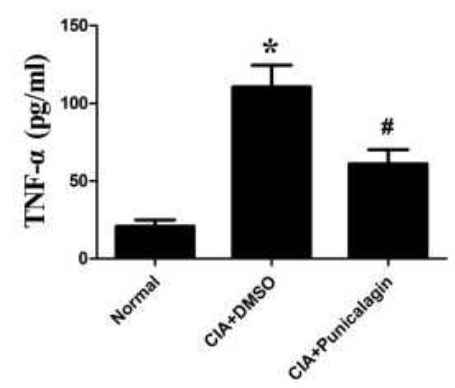

$\mathbf{F}$
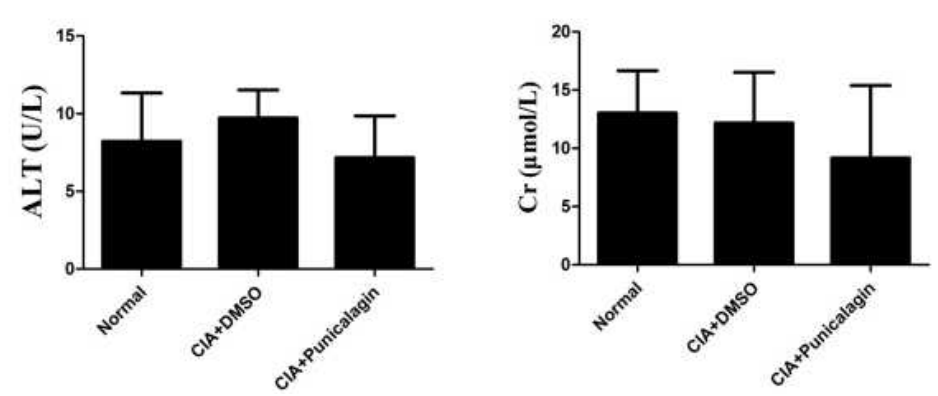

Figure 8 Punicalagin attenuated arthritis severity of CIA mice. Mice were treated with either dimethyl sulfoxide (DMSO; vehicle) or punicalagin (50 mg/kg/day) for I4 consecutive days. (A) H\&E staining in the upper panel was used to observe synovial infiltration, hyperplasia, and bone destruction $(\times 100)$. Safranin O/Fast green staining to proteoglycan depletion was shown in the lower panel (Original magnification $\times 100)$. The blue arrow represents inflammation infiltration, the black arrow represents bone erosion, the white arrow represents the hyperplasia. ${ }^{*} P<0.05$ vs Normal, ${ }^{\#} P<0.05$ vs CIA. (B-C) Punicalagin ameliorated clinical scores and paw swelling (change in paw thickness) of mice. Data represent the mean \pm SEM of 3 independent experiments. $* P<0.05$ vs DMSO. (D) Punicalagin decreased the serum levels of IL- 6 and TNF- $\alpha$ of CIA mice. The levels of the cytokines were measured by ELSIA. (E) Serum level of aspartate aminotransferase (AST) and alanine aminotransferase (ALT). (F) Serum level of creatinine (CRE), respectively. $* P<0.05$ vs normal control, ${ }^{\#} P<0.05$ vs DMSO.

Abbreviation: H\&E staining, hematoxylin and eosin staining. 
$\alpha$ induced phosphorylation of IKK $\beta$ and $\operatorname{I\kappa } \mathrm{B} \alpha$, and the intracellular translocation of $\mathrm{p} 65$, suggesting that punicalagin regulated NF- $\kappa$ B pathway activation via interfering in early IKK signaling pathway. Consistent with our data, previous studies have shown that punicalagin inhibited activation of NF- $\kappa$ B signal pathway in RAW264.7 macrophages, ${ }^{8}$ BCPAP cells, ${ }^{40}$ osteoblast cell lines. ${ }^{34}$ Our findings suggested that the regulations of $\mathrm{NF}-\kappa \mathrm{B}$ pathway was potentially involved in the beneficial effects of punicalagin.

CIA model is commonly used in the studies of RA. To confirm whether punicalagin have effect on RA in vivo, CIA mice received treatments of punicalagin $(50 \mathrm{mg} / \mathrm{kg} /$ day $)$ in our study. We demonstrated that punicalagin alleviated limb edema and swelling in CIA mice. Punicalagin decreased serum levels of both IL-6 and TNF- $\alpha$ in CIA mice. Pathological analysis indicated punicalagin treatments significantly attenuated the cellular infiltration, synovial hyperplasia, and cartilage destruction in CIA mice. Thus, our research demonstrated that punicalagin exert anti-arthritis effects of CIA.

To the best of our knowledge, this is the first study to report that punicalagin can exert a potent curative effect in CIA mice without obvious toxicity. We also confirmed that punicalagin had multiple effects on RA FLSs in vitro. However, it had its own limitations. More researches are needed to further explore the underlying mechanism of punicalagin in the treatment of RA. Moreover, studies are required in the human body after consumption of punicalagin from pomegranate juice, thus promote its future application.

\section{Conclusions}

Our findings provided new in vivo and in vitro evidences for the anti-arthritis effects of punicalagin in RA. We identified the inhibitory effects of punicalagin in the production of pro-inflammatory cytokines and MMPs by suppressing activations of NF- $\kappa \mathrm{B}$ pathway. Punicalagin also suppressed the proliferation and migration of RA FLSs. Moreover, punicalagin alleviated arthritis severity and bone destruction, and decreased serum levels of both IL6 and TNF- $\alpha$ in CIA mice. In summary, these results indicated that punicalagin was one of the natural therapeutic compounds to prevent and treat of RA.

\section{Acknowledgments}

This work was supported by National Natural Science Foundation of China (81603435, 81901647), Natural Science Foundation of Guangdong Province (2020A151 5010201) and grant from Young Teacher Foundation of Sun
Yat-sen University (19ykpy72 and 20ykpy16). Mingcheng Huang, Keping Wu, and Shan Zeng are co-first authors in this study.

\section{Disclosure}

The authors declare no conflicts of interest.

\section{References}

1. Falconer J, Murphy AN, Young SP, et al. Review: synovial cell metabolism and chronic inflammation in rheumatoid arthritis. Arthritis Rheumatol. 2018;70:984-999.

2. Bustamante MF, Garcia-Carbonell R, Whisenant KD, Guma M. Fibroblast-like synoviocyte metabolism in the pathogenesis of rheumatoid arthritis. Arthritis Res Ther. 2017;19:110.

3. Yoshitomi H. Regulation of immune responses and chronic inflammation by fibroblast-like synoviocytes. Front Immunol. 2019;10:1395.

4. Sajadimajd S, Bahramsoltani R, Iranpanah A, et al. Advances on natural polyphenols as anticancer agents for skin cancer. Pharmacol Res. 2020;151:104584.

5. Serino A, Salazar G. Protective role of polyphenols against vascular inflammation, aging and cardiovascular disease. Nutrients. 2018;11.

6. Mastrogiovanni F, Mukhopadhya A, Lacetera N, et al. Antiinflammatory effects of pomegranate peel extracts on in vitro human intestinal caco-2 cells and ex vivo porcine colonic tissue explants. Nutrients. 2019;11.

7. Cerda B, Ceron JJ, Tomas-Barberan FA, Espin JC. Repeated oral administration of high doses of the pomegranate ellagitannin punicalagin to rats for 37 days is not toxic. J Agric Food Chem. 2003;51:3493-3501.

8. Cao Y, Chen J, Ren G, Zhang Y, Tan X, Yang L. Punicalagin prevents inflammation in LPS-Induced RAW264.7 macrophages by inhibiting FOXO3A/autophagy signaling pathway. Nutrients. 2019;11.

9. Adams LS, Seeram NP, Aggarwal BB, Takada Y, Sand D, Heber D. Pomegranate juice, total pomegranate ellagitannins, and punicalagin suppress inflammatory cell signaling in colon cancer cells. J Agric Food Chem. 2006;54:980-985.

10. Seeram NP, Adams LS, Henning SM, et al. In vitro antiproliferative, apoptotic and antioxidant activities of punicalagin, ellagic acid and a total pomegranate tannin extract are enhanced in combination with other polyphenols as found in pomegranate juice. $J$ Nutr Biochem. $2005 ; 16: 360-367$.

11. Koyama S, Cobb LJ, Mehta HH, et al. Pomegranate extract induces apoptosis in human prostate cancer cells by modulation of the IGF-IGFBP axis. Growth Horm IGF Res. 2010;20:55-62.

12. Pan L, Duan Y, Ma F, Lou L. Punicalagin inhibits the viability, migration, invasion, and EMT by regulating GOLPH3 in breast cancer cells. J Recept Signal Transduct Res. 2020;40:173-180.

13. Jean-Gilles D, Li L, Vaidyanathan VG, et al. Inhibitory effects of polyphenol punicalagin on type-II collagen degradation in vitro and inflammation in vivo. Chem Biol Interact. 2013;205:90-99.

14. Li Y, Yang F, Zheng W, et al. Punica granatum (pomegranate) leaves extract induces apoptosis through mitochondrial intrinsic pathway and inhibits migration and invasion in non-small cell lung cancer in vitro. Biomed Pharmacother. 2016;80:227-235.

15. Zhang Q, Lenardo MJ, Baltimore D. 30 Years of NF-kappaB: a blossoming of relevance to human pathobiology. Cell. 2017;168:37-57.

16. Zou Y, Zeng S, Huang M, et al. Inhibition of 6-phosphofructo2-kinase suppresses fibroblast-like synoviocytes-mediated synovial inflammation and joint destruction in rheumatoid arthritis. $\mathrm{Br}$ J Pharmacol. 2017;174:893-908. 
17. Zou Y, Xu S, Xiao Y, et al. Long noncoding RNA LERFS negatively regulates rheumatoid synovial aggression and proliferation. $\mathrm{J} \mathrm{Clin}$ Invest. 2018;128:4510-4524.

18. Lao M, Shi M, Zou Y, et al. Protein inhibitor of activated STAT3 regulates migration, invasion, and activation of fibroblast-like synoviocytes in rheumatoid arthritis. J Immunol. 2016;196:596-606.

19. Li N, Xu Q, Liu Q, et al. Leonurine attenuates fibroblast-like synoviocyte-mediated synovial inflammation and joint destruction in rheumatoid arthritis. Rheumatology (Oxford). 2017;56:1417-1427.

20. Liang L, Huang M, Xiao Y, et al. Inhibitory effects of niclosamide on inflammation and migration of fibroblast-like synoviocytes from patients with rheumatoid arthritis. Inflamm Res. 2015;64:225-233.

21. Shi M, Wang J, Xiao Y, et al. Glycogen metabolism and rheumatoid arthritis: the role of glycogen synthase 1 in regulation of synovial inflammation via blocking AMP-activated protein kinase activation. Front Immunol. 2018;9:1714.

22. Ilchovska DD, Barrow DM. An overview of the NF-kB mechanism of pathophysiology in rheumatoid arthritis, investigation of the NF-kB ligand RANKL and related nutritional interventions. Autoimmun Rev. 2021;20:102741.

23. Rasines-Perea Z, Teissedre PL. Grape polyphenols' effects in human cardiovascular diseases and diabetes. Molecules. 2017;22.

24. McGrattan AM, McGuinness B, McKinley MC, et al. Diet and inflammation in cognitive ageing and Alzheimer's disease. Curr Nutr Rep. 2019;8:53-65.

25. Casas R, Castro-Barquero S, Estruch R, Sacanella E. Nutrition and cardiovascular health. Int J Mol Sci. 2018;19.

26. Chen Z, Bozec A, Ramming A, Schett G. Anti-inflammatory and immune-regulatory cytokines in rheumatoid arthritis. Nat Rev Rheumatol. 2019;15:9-17.

27. Russo RC, Garcia CC, Teixeira MM, Amaral FA. The CXCL8/IL-8 chemokine family and its receptors in inflammatory diseases. Expert Rev Clin Immunol. 2014;10:593-619.

28. Olajide OA, Kumar A, Velagapudi R, Okorji UP, Fiebich BL. Punicalagin inhibits neuroinflammation in LPS-activated rat primary microglia. Mol Nutr Food Res. 2014;58:1843-1851.

29. $\mathrm{Xu} \mathrm{X}$, Yin $\mathrm{P}$, Wan $\mathrm{C}$, et al. Punicalagin inhibits inflammation in LPS-induced RAW264 7 macrophages via the suppression of TLR4-mediated MAPKs and NF-Kappab activation. Inflammation. 2014;37:956-965.
30. Peng J, Wei D, Fu Z, et al. Punicalagin ameliorates lipopolysaccharide-induced acute respiratory distress syndrome in mice. Inflammation. 2015;38:493-499.

31. Yu LM, Dong X, Xue XD, et al. Protection of the myocardium against ischemia/reperfusion injury by punicalagin through an SIRT1-NRF-2-HO-1-dependent mechanism. Chem Biol Interact. 2019;306:152-162.

32. Seo Y, Mun $\mathrm{CH}$, Park $\mathrm{SH}$, et al. Punicalagin ameliorates lupus nephritis via inhibition of PAR2. Int J Mol Sci. 2020;21.

33. Feng $\mathrm{X}$, Yang $\mathrm{Q}$, Wang $\mathrm{C}$, Tong $\mathrm{W}, \mathrm{Xu} \mathrm{W}$. Punicalagin exerts protective effects against ankylosing spondylitis by regulating NF-kappaB-TH17/JAK2/STAT3 signaling and oxidative stress. Biomed Res Int. 2020;2020:4918239.

34. Wang XZ, Zhang SF, Yang ZH, Ye ZW, Liu J. Punicalagin suppresses osteosarcoma growth and metastasis by regulating NF-kappaB signaling. J Biol Regul Homeost Agents. 2020;34:1699-1708.

35. Tang JM, Min J, Li BS, et al. Therapeutic effects of punicalagin against ovarian carcinoma cells in association with beta-catenin signaling inhibition. Int J Gynecol Cancer. 2016;26:1557-1563.

36. Vincenti MP, Brinckerhoff CE. Transcriptional regulation of collagenase (MMP-1, MMP-13) genes in arthritis: integration of complex signaling pathways for the recruitment of gene-specific transcription factors. Arthritis Res. 2002;4:157-164.

37. Seok JK, Lee JW, Kim YM, Boo YC. Punicalagin and (-)-Epigallocatechin-3-gallate rescue cell viability and attenuate inflammatory responses of human epidermal keratinocytes exposed to airborne particulate matter PM10. Skin Pharmacol Physiol. 2018;31:134-143.

38. Elder SH, Mosher ML, Jarquin P, Smith P, Chironis A. Effects of short-duration treatment of cartilage with punicalagin and genipin and the implications for treatment of osteoarthritis. $J$ Biomed Mater Res B Appl Biomater. 2020.

39. Giridharan S, Srinivasan M. Mechanisms of NF-kappaB p65 and strategies for therapeutic manipulation. $J$ Inflamm Res. 2018;11:407-419.

40. Cheng X, Yao X, Xu S, et al. Punicalagin induces senescent growth arrest in human papillary thyroid carcinoma BCPAP cells via NF-kappaB signaling pathway. Biomed Pharmacother. 2018;103: 490-498.
Journal of Inflammation Research

\section{Publish your work in this journal}

The Journal of Inflammation Research is an international, peerreviewed open-access journal that welcomes laboratory and clinica findings on the molecular basis, cell biology and pharmacology of inflammation including original research, reviews, symposium reports, hypothesis formation and commentaries on: acute/chronic inflammation; mediators of inflammation; cellular processes; molecular mechanisms; pharmacology and novel anti-inflammatory drugs; clinical conditions involving inflammation. The manuscript management system is completely online and includes a very quick and fair peerreview system. Visit http://www.dovepress.com/testimonials.php to read real quotes from published authors. 